\title{
Biological safety in the medical laboratory
}

\author{
Janice Lo *, FRCPA, FHKCPath \\ Public Health Laboratory Centre, Department of Health, 382 Nam Cheong Street, Shek Kip Mei, Kowloon, Hong Kong
}

\begin{tabular}{l} 
Hong Kong Med J 2015;21:200 " janicelo@ \\
\hline DOI: 10.12809/hkmj154581 \\
Medical laboratories primarily process and perform
\end{tabular}

Medical laboratories primarily process and perform
testing on human specimens to provide results and interpretation for individual patient management, infection control, and public health purposes. Any clinical specimen potentially contains biological agents, such as viruses, bacteria, fungi, or parasites. It is therefore essential to ensure biological safety in the medical laboratory to prevent laboratory-acquired infections by laboratory staff and dissemination of any infectious agent from the laboratory.

Micro-organisms have generally been categorised into four Risk Groups, and medical laboratories are classified into four Biosafety Levels (BSLs). ${ }^{1}$ Each BSL has designated requirements in terms of architectural features and ventilation, safety equipment such as biological safety cabinets, use of personal protective equipment, and adoption of safe microbiological practices by qualified and trained personnel. Human specimens, which potentially contain human pathogens, are required to be handled at least at BSL-2. Pathogens that pose a high individual and community risk, with the potential to cause serious disease and that can be readily transmitted, with no effective treatment or preventive measures, are generally recommended to be handled with BSL-4 precautions. Nevertheless, such classifications, with only four levels, cannot be implemented mechanically. Risk assessment must incorporate various factors, such as the specific laboratory procedures and route of transmission of the pathogen.

In March 2014, the world was first alerted to the ongoing outbreak of Ebola virus disease (EVD) in West Africa. ${ }^{2}$ As of 19 April 2015, 26044 confirmed, probable, and suspected cases of EVD had been reported in the countries with widespread and intense transmission (namely in Guinea, Liberia, and Sierra Leone), with 10808 reported deaths. ${ }^{3}$ The Ebola virus, first described in 1976, has been generally regarded as a Risk Group 4 agent, and handling of live cultures needs to be undertaken at BSL-4. Globally, there are few BSL-4-certified laboratories, and such facilities require significant financial and human resources in order to operate effectively. Hong Kong does not have BSL-4 facilities. With the efficiency of modern travel, there is a possibility that a patient at the incubation stage of EVD (incubation period, 2 to 21 days) could arrive in Hong Kong and develop the disease. Medical laboratories in Hong Kong thus need to be prepared for supporting the diagnosis and management of EVD patients while ensuring laboratory safety. An article in this issue of the Hong Kong Medical Journal describes the effect of heating plasma specimens at $60^{\circ} \mathrm{C}$ for 60 minutes on the results of chemical pathology tests. ${ }^{4}$ The authors concluded that heat inactivation did not significantly affect electrolytes, glucose or renal function test results, but caused a significant bias for many analytes, especially enzymes. Thus for safety and diagnostic accuracy in suspected or confirmed cases of EVD, it was proposed to use pointof-care devices for blood gases, electrolytes, troponin, liver and renal function tests within a biosafety cabinet with BSL-3 practices.

Ebola virus can infect normal healthy persons, with a high fatality rate. There is no effective treatment or vaccine. Nevertheless it is not transmitted via inhalation but by direct contact of non-intact skin or mucous membranes with infected bodily fluids. As such, precautions against infection are targeted at prevention of exposure of nonintact skin or mucous membranes to the virus. In a diagnostic medical laboratory, nucleic acid testing is the investigation of choice to detect Ebola virus in clinical specimens, preferably blood, that are inactivated by extraction of nucleic acids prior to the test proper. Without the need for performing virus isolation, which yields high concentrations of the live virus, and based on the above principles and practical considerations, BSL-4 containment facilities should not be mandatory to undertake testing of specimens from EVD cases.

As elaborated above, while the medical laboratory must support patient diagnosis and management, as well as public health measures, it is essential to maintain biological safety in the laboratory to protect both the laboratory worker and the environment. This can only be achieved when quality standards in medical laboratories, in terms of facilities, equipment and specialist supervision, are duly enforced and continually maintained.

\section{References}

1. Laboratory biosafety manual. 3rd ed. Geneva: World Health Organization; 2004.

2. Ebola virus disease. April 2015. Available from: http:// www.who.int/mediacentre/factsheets/fs103/en/. Accessed 25 Apr 2015.

3. Ebola situation report. 22 April 2015. Available from: http://apps.who.int/iris/bitstream/10665/162795/1/ roadmapsitrep_22Apr2015_eng.pdf. Accessed 25 Apr 2015.

4. Chong YK, Ng WY, Chen SP, Mak CM. Effects of a plasma heating procedure for inactivating Ebola virus on common chemical pathology tests. Hong Kong Med J 2015;21:2017. 